\title{
ON VERBAL SUBGROUPS IN RESIDUALLY FINITE GROUPS
}

\author{
JHONE CALDEIRA and PAVEL SHUMYATSKY ${ }^{凶}$
}

\author{
(Received 30 December 2010)
}

\begin{abstract}
The following theorem is proved. Let $m, k$ and $n$ be positive integers. There exists a number $\eta=\eta(m, k, n)$ depending only on $m, k$ and $n$ such that if $G$ is any residually finite group satisfying the condition that the product of any $\eta$ commutators of the form $\left[x^{m}, y_{1}, \ldots, y_{k}\right]$ is of order dividing $n$, then the verbal subgroup of $G$ corresponding to the word $w=\left[x^{m}, y_{1}, \ldots, y_{k}\right]$ is locally finite.
\end{abstract}

2010 Mathematics subject classification: primary 20E10; secondary 20E26, 20F40, 20F50.

Keywords and phrases: residually finite groups, restricted Burnside problem, varieties.

\section{Introduction}

One immediate corollary of the solution of the restricted Burnside problem is that every residually finite group of finite exponent is locally finite. The solution of the problem for nilpotent groups was obtained by Zelmanov in [30, 31] while the reduction to the nilpotent case was carried out by Hall and Higman [11]. The reduction was based on certain conjectures about finite simple groups that were later confirmed by the classification.

The methods used in the solution happened to be very effective in treating other problems in group theory. In [22] the methods were used to prove the following theorem.

THEOREM 1.1. Let $n$ be a prime power and $G$ a residually finite group satisfying the identity $[x, y]^{n} \equiv 1$. Then $G^{\prime}$ is locally finite.

Note that in general a periodic residually finite group need not be locally finite. The corresponding examples have been constructed in [2, 7-9, 28]. Moreover, if the assumption that $G$ is residually finite is dropped from the hypothesis of Theorem 1.1, the derived group need not even be periodic. Deryabina and Kozhevnikov showed that for sufficiently large odd integers $n$ there exist groups $G$ in which all commutators have order dividing $n$ such that $G^{\prime}$ has elements of infinite order [4]. This was also proved independently by Adian [1]. Later Ivanov constructed similar examples for arbitrary

Supported by Capes and CNPq-Brazil.

(c) 2011 Australian Mathematical Publishing Association Inc. 0004-9727/2011 \$16.00 
sufficiently large integers $n$ [14]. In view of Theorem 1.1 the following problem was raised in [24].

PROBLEM 1.2. Let $n$ be a positive integer and $w$ a word. Assume that $G$ is a residually finite group such that any $w$-value in $G$ has order dividing $n$. Does it follow that the verbal subgroup $w(G)$ is locally finite?

At present the following is known with respect to the above problem.

(1) If $G$ is a residually finite group in which every product of 68 commutators has order dividing $n$, then $G^{\prime}$ is locally finite [26].

(2) If $w$ is a multilinear commutator and $G$ is a residually finite group in which every product of $896 w$-values has order dividing $n$, then $w(G)$ is locally finite [21].

(3) There exists $s$ depending only on $n$ and $k$ such that if $G$ is a residually finite group in which every product of $s$ values of the $k$ th Engel word is of order dividing $n$, then the verbal subgroup corresponding to the $k$ th Engel word is locally finite [27].

A word $w$ is called a multilinear commutator if it has form of a multilinear Lie monomial. In other terminology these words are called outer commutator words. Particular examples of multilinear commutators are the familiar derived words and the lower central words. An example of the commutator word which is not a multilinear commutator is provided by Engel words. Recall that Engel words are defined inductively by

$$
[x, 0 y]=x, \quad[x, k y]=[[x, k-1 y], y] .
$$

Until now the Engel words are the only nonmultilinear words for which reasonable progress with respect to Problem 1.2 has been achieved.

The aim of the present paper is to give a similar treatment for the case where $w=\left[x^{m}, y_{1}, \ldots, y_{k}\right]$. Theorem 6.2 in Section 6 says that there exists a number $\eta=\eta(m, k, n)$ depending only on $m, k$ and $n$ such that if $G$ is any residually finite group satisfying the condition that the product of any $\eta$ commutators of the form $\left[x^{m}, y_{1}, \ldots, y_{k}\right]$ is of order dividing $n$, then the verbal subgroup of $G$ corresponding to the word $w=\left[x^{m}, y_{1}, \ldots, y_{k}\right]$ is locally finite.

The general plan of the proof of the above result follows rather closely that of the main results in $[25,27]$ and, loosely, the same plan that led to the solution of the restricted Burnside problem.

\section{Notation and preliminary results}

We use the expression ' $\{a, b, c, \ldots\}$-bounded' to mean 'bounded from above by some function depending only on $a, b, c, \ldots$. Throughout the paper $m$ and $k$ stand for fixed positive integers. Having fixed $m$ and $k, w$ will denote the word $\left[x^{m}, y_{1}, \ldots, y_{k}\right]$. Given a group $G$, an element $g \in G$ will be called a $w$-value if there exist $x, y_{1}, \ldots, y_{k} \in G$ such that $g=\left[x^{m}, y_{1}, \ldots, y_{k}\right]$. The symbol $w_{j}$ will denote the word that is the product of $j w$-values. Thus, for example, the symbol $w_{2}$ 
will denote the word $\left[x_{1}^{m}, y_{1}, \ldots, y_{k}\right]\left[x_{2}^{m}, z_{1}, \ldots, z_{k}\right]$. It is clear that if an element of a group $G$ is a $w_{i}$-value, then it is also a $w_{j}$-value for any $i \leq j$. Therefore, for any $i \leq j$, the identity $w_{j}^{n} \equiv 1$ implies the identity $w_{i}^{n} \equiv 1$ in $G$. Obviously, the identity $w_{j}^{n} \equiv 1$ implies that all $w$-values have order dividing $n$, and this fact will be used freely without being explicitly mentioned.

The well-known Schur's theorem says that if the center $Z(G)$ of a group $G$ has finite index $m$, then $G^{\prime}$ is finite and has $m$-bounded order. Mann showed that for finite groups the following result of similar nature holds [18]: if $G$ is a finite group such that $G / Z(G)$ has exponent $m$, then the exponent of $G^{\prime}$ is $m$-bounded. Lemma 2.2 below is an easy extension of Mann's theorem. As usual, the symbol $Z_{k}(G)$ denotes the $k$ th term of the upper central series of a group $G$.

LEMMA 2.1. Let $G$ be a group such that $\left|G / Z_{k}(G)\right|=m$. Then the order of $\gamma_{k+1}(G)$ is $\{k, m\}$-bounded.

PROOF. Since $Z_{k}(G)$ is the marginal subgroup of the word $\gamma_{k+1}$, from the condition that $\left|G / Z_{k}(G)\right|=m$ one obtains that $\gamma_{k+1}$ takes at most $m^{k+1}$ values in $G$. It now follows from [3, Theorem 3.1] that the order of $\gamma_{k+1}(G)$ is $\{m, k\}$-bounded.

LEMMA 2.2. There exists $a\{k, m\}$-bounded number $e=e(k, m)$ such that if $G$ is a finite group with the property that $G / Z_{k}(G)$ has exponent $m$, then the exponent of $\gamma_{k+1}(G)$ divides $e$.

PROOF. In the case where $k=1$ this is Mann's theorem. Assume that $k \geq 2$ and use induction on $k$. Let $Z=Z(G)$. By induction the image of $\gamma_{k}(G)$ in $G / Z$ has bounded exponent. Therefore, by Mann's theorem, the derived group of $\gamma_{k}(G)$ has bounded exponent and we can pass to the quotient $G /\left[\gamma_{k}(G), \gamma_{k}(G)\right]$. Hence, without loss of generality, we assume that $\gamma_{k}(G)$ is abelian. Therefore it is sufficient to show that for every $x_{1}, \ldots, x_{k}, x_{k+1} \in G$ the commutator $x=\left[x_{1}, \ldots, x_{k}, x_{k+1}\right]$ has bounded order. We now can assume that $G=\left\langle x_{1}, \ldots, x_{k}, x_{k+1}\right\rangle$. Since $G / Z_{k}(G)$ has exponent $m$, it follows from the solution of the restricted Burnside problem that $G / Z_{k}(G)$ has bounded order. Now the result is immediate from the previous lemma.

It will be useful to remark that $G / Z_{k}(G)$ has exponent $m$ if and only if $G$ satisfies the identity $\left[x^{m}, y_{1}, \ldots, y_{k}\right] \equiv 1$. Having fixed $m$ and $k$, we write $e$ for $e(m, k)$ as in Lemma 2.2.

PROPOSITION 2.3. Let $p$ be a prime and $q$ a p-power. There exists a number $\mu=\mu(m, k, q)$ depending only on $m, k$ and $q$ with the following property: if $G$ is a finite p-group generated by $2 k$ elements, each of order dividing eq, and if $G$ satisfies the identity $w_{\mu}^{q} \equiv 1$, then $\gamma_{k+1}(G)$ has exponent dividing eq.

PROOF. Let $G$ be a finite $p$-group generated by $2 k$ elements, each of order dividing $e q$. Let $W$ be the verbal subgroup of $G$ corresponding to the word $w$. The quotient $G / W$ satisfies the identity $\left[x^{m}, y_{1}, \ldots, y_{k}\right] \equiv 1$ so, by Lemma $2.2, W$ is contained in a normal subgroup $S$ such that $G / S$ is nilpotent of class at most $k$ and $S / W$ is of 
exponent dividing $e$. As the orders of the generators of $G$ are bounded, it follows that $G / S$ has $\{m, k, q\}$-bounded order. Hence, the minimal number of generators of $S / W$ is $\{m, k, q\}$-bounded. By the solution of the restricted Burnside problem we conclude that $S / W$ has $\{m, k, q\}$-bounded order. Let $r$ be the minimal number of generators of $W$. It follows that the number $r$ is $\{m, k, q\}$-bounded. Applying Burnside's basis theorem [12, Ch. III, Section 3, Theorem 15], we derive that $W$ is generated by $r w$-values $b_{1}, \ldots, b_{r}$, say. Any element of $W^{\prime}$ can be written in the form $\left[x_{1}, b_{1}\right] \cdots\left[x_{r}, b_{r}\right]$ for suitable $x_{1}, \ldots, x_{r} \in W[5$, Lemma 1.23]. We now put $\mu=q r+q^{r}$ and assume that $G$ satisfies the identity $w_{\mu}^{q} \equiv 1$. Since each one of the commutators $\left[x_{i}, b_{i}\right]$ is a product of a $w$-value and of the inverse of a $w$-value and since the inverse is the $(q-1)$ th power of a $w$-value, we conclude that any element of $W^{\prime}$ is a product of at most $q r w$-values. As $W / W^{\prime}$ is generated by $w$-values and has order at most $q^{r}$, it follows that any element of $W$ is a product of at most $\mu w$-values. In particular, the exponent of $W$ is at most $q$, whence that of $S$ is at most $e q$. It remains to remark that $\gamma_{k+1}(G) \leq S$.

\section{A result about finite $p$-groups}

According to the solution of the restricted Burnside problem the order of any finite $r$-generated group of exponent $n$ is $\{r, n\}$-bounded. The goal of this section is to prove the following related result.

Proposition 3.1. Let $\mu=\mu(m, k, q)$ be as in Proposition 2.3. Let $l$ be a positive integer and $G$ a finite p-group generated by $r$ elements $a_{1}, \ldots, a_{r}$, each of order dividing $l$. Suppose that $G$ satisfies the identity $w_{\mu}^{q} \equiv 1$. Then the order of $G$ is $\{m, k, q, r, l\}$-bounded.

The proof of the above proposition is based on Lie methods in the spirit of Zelmanov's solution of the restricted Burnside problem. For the reader's convenience we collect some definitions and facts on Lie algebras associated with $p$-groups.

Let $L$ be a Lie algebra over a field $\mathbb{F}$. We use the left normed notation

$$
\left[x_{1}, x_{2}, x_{3}, \ldots, x_{r}\right]=\left[\cdots\left[\left[x_{1}, x_{2}\right], x_{3}\right], \ldots, x_{r}\right]
$$

for all $x_{1}, x_{2}, x_{3}, \ldots, x_{r} \in L$. An element $y \in L$ is called ad-nilpotent if there exists a positive integer $r$ such that

$$
[x, \underbrace{y, \ldots, y}_{r}]=0 \quad \text { for all } x \in L .
$$

If $r$ is the least positive integer with the above property, then we say that $y$ is adnilpotent of index $r$.

If $X \subseteq G$ is any subset of a group $G$, by a commutator in elements of $X$ we mean any element of $G$ that could be obtained from elements of $X$ by means of repeated operation of commutation with an arbitrary system of brackets including the elements 
of $X$. In particular, the elements of $X$ are viewed as commutators with the empty system of brackets.

Let $G$ be a finite $p$-group. The terms of the lower central series of $G$ will be denoted by $\gamma_{j}(G)$. Write

$$
D_{i}=D_{i}(G)=\prod_{j \cdot p^{k} \geq i} \gamma_{j}(G)^{p^{k}} .
$$

The subgroups $D_{i}$ form a central series of $G$ known as the Zassenhaus-JenningsLazard series (see [13, Ch. 8]). Set $L(G)=\bigoplus D_{i} / D_{i+1}$. Then $L(G)$ can naturally be viewed as a Lie algebra over the field $\mathbb{F}_{p}$ with $p$ elements. In fact $L(G)$ even has the structure of a restricted Lie algebra (Lie $p$-algebra), but we will treat it as just a Lie algebra. We will denote by $L_{p}(G)$ the subalgebra of $L(G)$ generated by $D_{1} / D_{2}$. Fix a positive number $c$ and assume that $G$ is generated by $a_{1}, \ldots, a_{r}$. Let $\rho_{1}, \ldots, \rho_{s}$ be the list of all commutators in $a_{1}, \ldots, a_{r}$ of weight at most $c$. Here $s$ obviously is $\{r, c\}$-bounded. The following lemma is implicit in Zelmanov [32, p. 71]. A detailed proof can be found in [23, Proposition 2.11].

LEMMA 3.2. If $L_{p}(G)$ is nilpotent of class $c$ then the group $G$ can be written as a product $G=\left\langle\rho_{1}\right\rangle\left\langle\rho_{2}\right\rangle \cdots\left\langle\rho_{s}\right\rangle$ of cyclic subgroups generated by the $\rho_{j}$.

Let $x \in G$ and let $i=i(x)$ be the largest integer such that $x \in D_{i}$. We denote by $\tilde{x}$ the element $x D_{i+1} \in L(G)$.

LEMMA 3.3 (Lazard [17]). For any $x \in G$ we have $(a d \tilde{x})^{p}=\operatorname{ad}\left(\widetilde{x^{p}}\right)$. In particular, if $x^{q}=1$ then $\tilde{x}$ is ad-nilpotent of index at most $q$.

Any group law that holds in $G$ implies certain polynomial identity in the algebra $L_{p}(G)$. In Wilson and Zelmanov [29, Theorem 1] one can find a description of an effective algorithm allowing the polynomial identity to be written explicitly when the group law is given. Thus, we have the following lemma.

LEMMA 3.4. Let $G$ be a group satisfying an identity $v \equiv 1$. Then there exists a nonzero Lie polynomial $f$ whose form depends only on $p$ and $v$ such that the algebra $L_{p}(G)$ satisfies the identity $f \equiv 0$.

In view of Lemma 3.2, it is important to have criteria for a Lie algebra to be nilpotent of bounded class. The following result was proved in [16, Corollary of Theorem 4] using a profound theorem of Zelmanov [32, III(0.4)].

THEOREM 3.5. Let $L$ be a Lie algebra over $\mathbb{F}_{p}$ generated by $r$ elements $a_{1}, \ldots, a_{r}$. Assume that $L$ satisfies the identity $f \equiv 0$ and that each monomial in the generators $a_{1}, \ldots, a_{r}$ is ad-nilpotent of index at most $q$. Then $L$ is nilpotent of $\{f, r, q\}$-bounded class. 
We are now in a position to prove Proposition 3.1.

Proof of Proposition 3.1. Let $W$ be the verbal subgroup of $G$ corresponding to the word $w$. The group $G / W$ satisfies the identity $\left[x^{m}, y_{1}, \ldots, y_{k}\right] \equiv 1$. Therefore, by Lemma 2.2, $G / W$ is an extension of a group of exponent dividing $e$ by a nilpotent group of class at most $k$. The positive solution of the restricted Burnside problem now allows us to conclude that $G / W$ has $\{m, k, r, l\}$-bounded order. Hence, it is sufficient to prove that the order of $W$ is $\{m, k, q, r, l\}$-bounded. Proposition 2.3 tells us that if $x_{1}, \ldots, x_{2 k} \in W$ are any elements of order dividing $e q$, then $\gamma_{k+1}\left(\left\langle x_{1}, \ldots, x_{2 k}\right\rangle\right)$ has exponent dividing $e q$. Let

$$
S=\left\{\left[x_{1}, \ldots, x_{d}\right]: k+1 \leq d \leq 2 k ; x_{1}, \ldots, x_{d} \in W ; x_{1}^{e q}=\cdots=x_{d}^{e q}=1\right\} .
$$

It is clear that $S$ consists of elements of order dividing eq. We will now show that $[x, y] \in S$ whenever $x, y \in S$. Suppose that $x=\left[x_{1}, \ldots, x_{d}\right]$. If $d \leq 2 k-1$, then we write $[x, y]=\left[x_{1}, \ldots, x_{d}, y\right]$, and this clearly belongs to $S$. If $d=2 k$, set $z=\left[x_{1}, \ldots, x_{k+1}\right]$. Now write $[x, y]=\left[z, x_{k+2}, \ldots, x_{2 k}, y\right]$. Written in this way, the commutator has weight $k+1$ with all entries of order dividing $e q$. Thus, in all cases $[x, y] \in S$.

Let $T=\langle S\rangle$ be the subgroup of $W$ generated by the set $S$. Since $W$ is generated by elements of order dividing $q$, it follows that $\gamma_{k+1}(W) \leq T$. We know that the index of $W$ in $G$ is $\{m, k, r, l\}$-bounded. Therefore so is the minimal number of generators of $W$. We conclude that the proof of the proposition will be complete once it is shown that the order of $T$ is $\{m, k, q, l, r\}$-bounded.

Note that if $t$ is the minimal number of generators of $T$, then $t$ is $\{m, k, q, l, r\}$ bounded. Applying Burnside's basis theorem, we derive that $T$ is generated by some $t$ elements $b_{1}, \ldots, b_{t} \in S$.

We know that the identity $\left[x^{m}, y_{1}, \ldots, y_{k}\right]^{q} \equiv 1$ holds in $T$, so it follows by Lemma 3.4 that there exists a nonzero Lie polynomial $f$ over $\mathbb{F}_{p}$, whose form depends only on $m, k$ and $q$, such that the algebra $L_{p}(T)$ satisfies the identity $f \equiv 0$.

Consider an arbitrary Lie monomial $\sigma$ in the generators $\tilde{b}_{1}, \ldots, \widetilde{b}_{t}$ of $L_{p}(T)$ and let $\rho$ be the group commutator in $b_{1}, \ldots, b_{t}$ having the same arrangement of brackets as $\sigma$. The definition of $L_{p}(T)$ yields that either $\sigma=0$ or $\sigma=\widetilde{\rho}$. Since $\rho^{e q}=1$, Lemma 3.3 implies that $\sigma$ is ad-nilpotent of index at most eq. Theorem 3.5 now says that $L_{p}(T)$ is nilpotent of class depending only on $m, k, t$ and $q$. Combining this with Lemma 3.2, we conclude that there exists an $\{m, k, q, r, l\}$-bounded number $s$ such that $T$ can be written as a product of at most $s$ cyclic subgroups, each of order at most $e q$. Therefore $T$ is of order at most $e^{s} q^{s}$, as required.

\section{Bounding the Fitting height of a soluble group}

Recall that the Fitting subgroup $F(G)$ of $G$ is the product of all normal nilpotent subgroups of $G$. The Fitting series of $G$ can be defined by the rules: $F_{0}(G)=1$, $F_{1}(G)=F(G), F_{i+1}(G) / F_{i}(G)=F\left(G / F_{i}(G)\right)$ for $i=1,2, \ldots$ If $G$ is a finite 
soluble group, then the minimal number $h=h(G)$ such that $F_{h}(G)=G$ is called the Fitting height of $G$. We will require the following proposition.

Proposition 4.1. Let $G$ be a finite group and $x \in G$. Suppose that every subgroup of $G$ that can be generated by four conjugates of $x$ is soluble with Fitting height at most $h$. Then $x \in F_{h}(G)$.

Essentially, the above proposition is due to Flavell et al. [6]. A detailed proof can be found in [21].

In the proof of Theorem 6.2 we need to consider subgroups of a finite soluble group that can be generated by four $w$-values. Segal showed that every element in the derived group of a finite soluble $d$-generated group is a product of at most $72 d^{2}+46 d$ commutators [20]. In a later paper Nikolov and Segal obtained a better bound. Working through the proofs given in [19], one deduces that every element of the derived group of a finite soluble $d$-generated group is a product of at most

$$
\min \left\{d\left(6 d^{2}+3 d+4\right), 8 d(3 d+2)\right\}
$$

commutators. In the case where $d=4$ this is 448 .

A well-known corollary of the Hall-Higman theory says that the Fitting height of any finite soluble group of exponent $n$ is bounded in terms of the number of prime divisors of $n$, counting multiplicities. We will denote the bound by $h(n)$.

LEMMA 4.2. Let $j$ and $n$ be positive integers with the property that $j \geq 448 n+n^{4}$. Let $G$ be a finite soluble group satisfying the identity $w_{j}^{n} \equiv 1$. Then the Fitting height of the verbal subgroup $w(G)$ corresponding to the word $w$ is at most $h(n)$.

Proof. Set $h=h(n)$. It is sufficient to prove that any $w$-value in $G$ belongs to $F_{h}(G)$. Given a $w$-value $v \in G$, let $H$ be the subgroup of $G$ generated by four conjugates of $v$. If $H$ has Fitting height at most $h$ then $v \in F_{h}(G)$. Now we apply Proposition 4.1.

Let $a_{1}$ be a $w$-value in $G$ and $a_{2}, a_{3}, a_{4}$ conjugates of $a_{1}$. Put $H=\left\langle a_{1}, a_{2}, a_{3}, a_{4}\right\rangle$. Since conjugates of a $w$-value are again $w$-values, each of the elements $a_{i}$ has order dividing $n$. We know from [19] that every element of $H^{\prime}$ can be written as a product of 448 commutators of the form $\left[b_{i}, c_{i}\right]$, where $b_{i} \in H$ and $c_{i} \in\left\{a_{1}, a_{2}, a_{3}, a_{4}\right\}$. We can write each of them as $c_{i}^{(n-1) b_{i}} c_{i}$. So if $x \in H^{\prime}$ then $x$ is a product of at most $448 n$ conjugates of $a_{i}$. Since $H / H^{\prime}$ is generated by elements of order dividing $n$ and the order of $H / H^{\prime}$ is at most $n^{4}$, it follows that any element of $H$ is a product of at most $448 n+n^{4}$ conjugates of $a_{i}$. By the hypothesis any such product has order dividing $n$. So $H$ is of exponent $n$, whence $h(H) \leq h$.

COROLlaRY 4.3. Under the hypothesis of Lemma 4.2, we have $h(G) \leq h(n)+h(m)$.

PROOF. Let $W$ is the verbal subgroup of $G$ corresponding to the word $w$. By the previous lemma, $h(W) \leq h(n)$. The quotient $\bar{G}=G / W$ satisfies the identity $\left[x^{m}, y_{1}, \ldots, y_{k}\right] \equiv 1$. So $\bar{G} / Z_{k}(\bar{G})$ has exponent $m$. The result follows. 


\section{Bounding the order of a finite group}

Following the terminology used by Hall and Higman [11] we call a finite group $G$ monolithic if it has a unique minimal normal subgroup which is nonabelian simple. In the modern literature such groups are very often called 'almost simple'.

PROPOSITION 5.1. Let $j, j_{1}$ and $n$ be positive integers with the property that $j \geq$ $j_{1} n+1$ and let $G$ be a finite group satisfying the identity $w_{j}^{n} \equiv 1$. Assume that $G$ has no nontrivial normal soluble subgroups. Then $G$ possesses a normal subgroup $L$ such that $L$ is residually monolithic and $G / L$ residually belongs to the class of finite groups satisfying an identity $w_{j_{1}}^{n / p} \equiv 1$ for some prime divisor $p$ of $n$.

Proof. Let $M$ be a minimal normal subgroup of $G$. We know that $M \cong S_{1} \times S_{2} \times$ $\cdots \times S_{r}$, where $S_{1}, S_{2}, \ldots, S_{r}$ are isomorphic simple groups. The group $G$ acts on $M$ by permuting the simple factors, so we obtain a representation of $G$ by permutations of the set $\left\{S_{1}, S_{2}, \ldots, S_{r}\right\}$. Let $L_{M}$ be the kernel of the representation. Choose in $S_{1}$ a nontrivial element $b$ of the form $w_{1}$. This is possible because $S_{1}$ is not soluble. Let $p$ be a prime divisor of the order of $b$. We want to show that $G / L_{M}$ satisfies the identity $w_{j_{1}}^{n / p} \equiv 1$. Suppose that this is not true. Let $q=p^{\alpha}$ be the largest power of $p$ dividing $n$. Since the identity $w_{j_{1}}^{n / p} \equiv 1$ does not hold in $G / L_{M}$, there exists an element $a \in G$ of the form $w_{j_{1}}$ such that $q$ divides the order of $a$ modulo $L_{M}$. Write $n=n_{1} q$ and $a_{1}=a^{n_{1}}$. Then $a_{1}$ is an element of the form $w_{j_{1} n_{1}}$ that has order $q$ and permute regularly some $q$ factors in $\left\{S_{1}, S_{2}, \ldots, S_{r}\right\}$. With no loss of generality, we will assume that $S_{1}$ is one of those factors. Write

$$
\left(b a_{1}\right)^{q}=b b^{a_{1}^{-1}} b^{a_{1}^{-2}} \cdots b^{a_{1}} .
$$

Since each of the elements $b^{a_{1}^{-i}}$ belongs to a different subgroup $S_{i}$, the product $b b^{a_{1}^{-1}} b^{a_{1}^{-2}} \cdots b^{a_{1}}$ has the same order as $b$. Thus, $\left(b a_{1}\right)^{q}$ has order divisible by $p$ so the order of $b a_{1}$ is divisible by $p^{\alpha+1}$. However, $b a_{1}$ is of the form $w_{j}$ so its order must divide $n$. This contradiction shows that $G / L_{M}$ does indeed satisfy the identity $w_{j_{1}}^{n / p} \equiv 1$.

Now let $L$ be the intersection of all the subgroups $L_{M}$, where $M$ ranges through the minimal normal subgroups of $G$. The previous paragraph implies that the proof of the proposition will be completed once it is shown that $L$ is residually monolithic. If $T$ is the product of the minimal normal subgroups of $G$, it is clear that $T$ is the product of pairwise commuting simple groups $S_{1}, S_{2}, \ldots, S_{t}$ and that $L$ is the intersection of the normalizers of $S_{i}$. Since $G$ has no nontrivial normal soluble subgroups, it follows that $C_{G}(T)=1$ and therefore any element of $L$ induces a nontrivial automorphism of some the $S_{i}$. Let $\rho_{i}$ be the natural homomorphism of $L$ into the group of automorphisms of $S_{i}$. It is easy to see that the image of $\rho_{i}$ is monolithic and that the intersection of the kernels of all $\rho_{i}$ is trivial. Hence, $L$ is residually monolithic.

Let $\mu=\mu(m, k, n)$ have the same meaning as in Proposition 2.3. Let us choose a function $\eta(x, y, z)$ defined for positive integers $x, y, z$ with the following properties. 
(1) $\quad \eta(x, y, z) \geq 448 z+z^{4}$ for all $x, y, z$.

(2) $\quad \eta(x, y, z) \geq \mu(x, y, z)$ whenever $z$ is a prime power.

(3) $\quad \eta(x, y, z) \geq z \cdot \eta(x, y, t)+1$ for all $x, y, z, t$ such that $t$ is a proper divisor of $z$.

Such a function can be constructed using induction on $z$. Indeed, fix a pair of positive integers $x$ and $y$ and define $\eta(x, y, 1)$ to be the maximum of the numbers 449 and $\mu(x, y, 1)$. Now suppose that $\eta(x, y, z)$ is defined for all $z \leq n-1$. If $n$ is a prime power, put $\eta(x, y, n)=\mu(x, y, n)$. Otherwise, let $M$ be the maximal value among $448 n+n^{4}$ and $n \cdot \eta(x, y, t)+1$, where $t$ ranges through the set of all proper divisors of $n$. Put $\eta(x, y, n)=M$. This can be done for any pair $x$ and $y$, thus establishing the existence of a function with the desired properties. Eventually, it will be shown that the chosen function satisfies the hypothesis of Theorem 6.2.

Proposition 5.2. Let $m, k, n, l$ be positive integers and $\eta=\eta(m, k, n)$. Let $G$ be a finite group satisfying the identity $w_{\eta}^{n} \equiv 1$. Assume that $G$ can be generated by $r$ elements $g_{1}, \ldots, g_{r}$ such that each $g_{i}$ and each commutator of the form $[g, x]$, where $g \in\left\{g_{1}, \ldots, g_{r}\right\}$ and $x \in G$, have order dividing $l$. Then the order of $G$ is $\{m, k, n, l, r\}$-bounded.

PROOF. If $n=1, G$ is a finite group satisfying $\left[x^{m}, y_{1}, \ldots, y_{k}\right] \equiv 1$. By Lemma 2.2, there exists a number $e=e(m, k)$ depending only on $m$ and $k$ with the property that $G$ has a normal subgroup $N$ such that $N$ is of exponent dividing $e$ and $G / N$ is a nilpotent group of class at most $k$. It is easy to see that $G / N$ has $\{m, k, n, l, r\}$-bounded order. The minimal number of generators of $N$ is bounded in terms of $r$ and $|G: N|$. The positive solution of the restricted Burnside problem allows us to conclude that $|N|$ and, therefore, $|G|$ are $\{m, k, n, l, r\}$-bounded.

We will now use induction on $n$. The case $n=1$ having been covered in the previous paragraph, suppose that $n \geq 2$ and that the proposition is true for groups satisfying an identity $w_{\eta(m, k, n / p)}^{n / p} \equiv 1$ for a prime divisor $p$ of $n$. In other words, the induction hypothesis is that there exists an $\{m, k, n, l, r\}$-bounded number $N_{0}$ such that if $G$ is a finite group satisfying the identity $w_{\eta(m, k, n / p)}^{n / p} \equiv 1$ that can be generated by $r$ elements $g_{1}, \ldots, g_{r}$ such that each $g_{i}$ and each commutator of the form $[g, x]$ have order dividing $l$, then $|G| \leq N_{0}$.

Suppose for a moment that $G$ has no nontrivial normal soluble subgroups. Since $\eta(m, k, n) \geq n \cdot \eta(m, k, n / p)+1$, Proposition 5.1 tells us that $G$ possesses a normal subgroup $L$ such that $L$ is residually monolithic and $G / L$ residually belongs to the class of finite groups satisfying an identity $w_{\eta(m, k, n / p)}^{n / p} \equiv 1$ for some prime divisor $p$ of $n$. It follows that $G / L$ is residually of order at most $N_{0}$. Since $G / L$ is $r$-generated, by [10, Theorem 7.2.9], the number of normal subgroups of index at most $N_{0}$ in $G / L$ is $\left\{r, N_{0}\right\}$-bounded. Therefore $|G / L|$ is $\{m, k, n, l, r\}$-bounded. In particular, it follows that $L$ can be generated by $t$ elements for some $\{m, k, n, l\}$-bounded number $t$.

A result of Jones [15] says that any infinite family of finite simple groups generates the variety of all groups. It follows that up to isomorphism there exist only finitely many monolithic groups satisfying the identity $w_{\eta}^{n} \equiv 1$. Let $N_{1}=N_{1}(n, \eta)$ be the 
maximum of their orders. Then $L$ is residually of order at most $N_{1}$. Since $L$ is $t$-generated, the number of distinct normal subgroups of index at most $N_{1}$ in $L$ is $\left\{t, N_{1}\right\}$-bounded. Therefore $L$ has $\{m, k, n, l, r\}$-bounded order. We conclude that $|G|$ is $\{m, k, n, l, r\}$-bounded.

Now let us drop the assumption that $G$ has no nontrivial normal soluble subgroups. Let $S$ be the product of all normal soluble subgroups of $G$. The above paragraph shows that $G / S$ has $\{m, k, n, l, r\}$-bounded order. Since $\eta(m, k, n) \geq 448 n+n^{4}$, by Corollary 4.3 the Fitting height of $S$ is $\{m, k, n, l, r\}$-bounded. Let $F=F(G)$ be the Fitting subgroup of $G$. Using induction on the Fitting height of $S$, we assume that $F$ has $\{m, k, n, l, r\}$-bounded index in $G$.

Suppose first that $F$ is central. In this case, $|G: Z(G)|$ is $\{m, k, n, l, r\}$-bounded and Schur's theorem guarantees that so is $\left|G^{\prime}\right|$. Since $G$ can be generated by $r$ elements of order dividing $l$, it follows that $|G|$ is $\{m, k, n, l, r\}$-bounded.

If $F$ is not central, consider the subgroup

$$
N=\left\langle\left[g_{1}, F\right], \ldots,\left[g_{r}, F\right]\right\rangle .
$$

It is easy to see that $N$ is normal in $G$. Applying the results of the previous paragraph to the quotient $G / N$, it follows that $|G: N|$ is $\{m, k, n, l, r\}$-bounded. We will show that $|N|$, and therefore also $|G|$, are $\{m, k, n, l, r\}$-bounded.

We know that $N$ can be generated by an $\{m, k, n, l, r\}$-bounded number of elements. Let $d$ be the minimal number of generators of $N$. Denote by $\pi(N)$ the set of prime divisors of $|N|$. Since $N$ is nilpotent, $\pi(N)$ consists of prime divisors of $l$. Thus, it is sufficient to bound the order of the Sylow $p$-subgroup of $N$ for every prime $p \in \pi(N)$. Let $P$ be the Sylow $p$-subgroup of $N$ and write $N=P \times O_{p^{\prime}}(N)$. If $y_{1}, y_{2}, \ldots$ is the list of all elements of the form $\left[g_{i}, y\right]$, where $1 \leq i \leq r$ and $y \in F$, we write $b_{1}, b_{2}, \ldots$ for the corresponding projections of $y_{j}$ in $P$. Then $P=\left\langle b_{1}, b_{2}, \ldots\right\rangle$. Since $P$ is a finite $d$-generated $p$-group, Burnside's basis theorem shows that $P$ is actually generated by $d$ elements in the list $b_{1}, b_{2}, \ldots$ By the hypothesis, the order of each of these divides $l$. Let $q$ be the maximal power of $p$ dividing $n$. Since $\eta(m, k, n) \geq \mu(m, k, q)$, by Proposition 3.1 we conclude that $P$ has $\{m, k, n, l, r\}$ bounded order. The proof is complete.

\section{Main results}

Recall that variety is a class of groups defined by equations. More precisely, if $W$ is a set of words in $x_{1}, x_{2}, \ldots$, the class of all groups $G$ such that $W(G)=1$ is called the variety determined by $W$. By a well-known theorem of Birkhoff, varieties are precisely classes of groups closed with respect to taking quotients, subgroups and Cartesian products of their members.

Theorem 6.1. Let $\eta=\eta(m, k, n)$ be as in Proposition 5.2. Let $\mathfrak{X}$ denote the class of all groups with the identity $w_{\eta}^{n} \equiv 1$ and the verbal subgroup $w(G)$ corresponding to the word $w$ locally finite. Then $\mathfrak{X}$ is a variety. 
PROOF. It is easy to see that the class $\mathfrak{X}$ is closed to taking subgroups and quotients of its members. Hence, we only need to show that if $D$ is a Cartesian product of groups from $\mathfrak{X}$, then $D \in \mathfrak{X}$. Obviously, the identity $w_{\eta}^{n} \equiv 1$ holds in $D$ so it remains only to show that the verbal subgroup $W$ of $D$ corresponding to the word $w$ is locally finite. Let $S$ be any finite subset of $W$. Clearly one can find finitely many $w$-values $h_{1}, \ldots, h_{s} \in D$ such that $S \leq\left\langle h_{1}, \ldots, h_{s}\right\rangle=H$. Thus it is sufficient to prove that the subgroup $H$ is finite. The order of each $h_{i}$ divides $n$. Moreover, if $h \in\left\{h_{1}, \ldots, h_{s}\right\}$ and $x \in H$, then each commutator of the form $[h, x]$ is a product of at most $n w$-values. It is clear from the choice of $\eta$ that $\eta(m, k, n) \geq n$ for any $n \geq 2$. So the order of each of the commutators divides $n$. Note that $W$ is residually locally finite. If $Q$ is any locally finite quotient of $W$, by Proposition 5.2 the order of the image of $H$ in $Q$ is finite and $\{m, k, n, s\}$-bounded, so it follows that this order actually does not depend on $Q$. We conclude that $H$ is finite, as required.

THEOREM 6.2. There exists a number $\eta=\eta(m, k, n)$ depending only on $m, k$ and $n$ such that if $G$ is any residually finite group satisfying the condition that the product of any $\eta$ commutators of the form $\left[x^{m}, y_{1}, \ldots, y_{k}\right]$ is of order dividing $n$, then the verbal subgroup of $G$ corresponding to the word $w=\left[x^{m}, y_{1}, \ldots, y_{k}\right]$ is locally finite.

PROOF. Let $\mathfrak{X}$ have the same meaning as in Theorem 6.1 and let $G$ satisfy the hypothesis of the theorem. Then $G$ residually belongs to $\mathfrak{X}$. Now, Theorem 6.1 tells us that $\mathfrak{X}$ is a variety. It follows that actually $G \in \mathfrak{X}$, that is, the verbal subgroup of $G$ corresponding to the word $w$ is locally finite.

\section{References}

[1] S. I. Adian, 'On groups with periodic commutators', Dokl. Math. 62 (2000), 174-176.

[2] S. V. Aleshin, 'Finite automata and the Burnside problem for periodic groups', Math. Notes 11 (1972), 199-203.

[3] S. Brazil, A. Krasilnikov and P. Shumyatsky, 'Groups with bounded verbal conjugacy classes', J. Group Theory 9 (2006), 127-137.

[4] G. S. Deryabina and P. A. Kozhevnikov, 'The derived subgroup of a group with commutators of bounded order can be nonperiodic', Comm. Algebra 27(9) (1999), 4525-4530.

[5] J. D. Dixon, M. P. F. du Sautoy, A. Mann and D. Segal, Analytic Pro-p Groups (Cambridge University Press, Cambridge, 1991).

[6] P. Flavell, S. Guest and R. Guralnick, 'Characterizations of the solvable radical', Proc. Amer. Math. Soc. 138 (2010), 1161-1170.

[7] E. S. Golod, 'On nil-algebras and residually finite groups', Izv. Akad. Nauk SSSR Ser. Mat. 28 (1964), 273-276.

[8] R. I. Grigorchuk, 'On the Burnside problem for periodic groups', Funct. Anal. Appl. 14 (1980), 53-54.

[9] N. Gupta and S. Sidki, 'On the Burnside problem for periodic groups', Math. Z. 182 (1983), 385-386.

[10] M. Hall, The Theory of Groups (Macmillan, New York, 1959).

[11] P. Hall and G. Higman, 'The $p$-length of a $p$-soluble group and reduction theorems for Burnside's problem', Proc. Lond. Math. Soc. 6(3) (1956), 1-42.

[12] B. Huppert, Endliche Gruppen I (Springer, Berlin, 1967). 
[13] B. Huppert and N. Blackburn, Finite Groups II (Springer, Berlin, 1982).

[14] S. Ivanov, 'On groups with periodic products of commutators', in: Topological and Asymptotic Aspects of Group Theory, Contemporary Mathematics, 394 (American Mathematical Society, Providence, RI, 2006), pp. 143-147.

[15] G. A. Jones, 'Varieties and simple groups', J. Aust. Math. Soc. 17 (1974), 163-173.

[16] E. I. Khukhro and P. Shumyatsky, 'Bounding the exponent of a finite group with automorphisms', J. Algebra 212 (1999), 363-374.

[17] M. Lazard, 'Sur les groupes nilpotents et les anneaux de Lie', Ann. Sci. Éc. Norm. Supér. 71 (1954), 101-190.

[18] A. Mann, 'The exponents of central factor and commutator groups', J. Group Theory 10(4) (2007), 435-436.

[19] N. Nikolov and D. Segal, 'On finitely generated profinite groups. I: Strong completeness and uniform bounds', Ann. of Math. (2) $\mathbf{1 6 5}(2)$ (2007), 171-238.

[20] D. Segal, 'Closed subgroups of profinite groups', Proc. Lond. Math. Soc. 81(3) (2000), 29-54.

[21] P. Shumyatsky, Multilinear commutators in residually finite groups, Israel J. Math., to appear, http://arxiv.org/abs/1012.2840.

[22] P. Shumyatsky, 'On groups with commutators of bounded order', Proc. Amer. Math. Soc. 127 (1999), 2583-2586.

[23] P. Shumyatsky, 'Applications of Lie ring methods to group theory', in: Nonassociative Algebra and its Applications (eds. R. Costa, A. Grishkov, H. Guzzo Jr and L. A. Peresi) (Marcel Dekker, New York, 2000), pp. 373-395.

[24] P. Shumyatsky, 'Verbal subgroups in residually finite groups', Q. J. Math. (Oxford) 51 (2000), 523-528.

[25] P. Shumyatsky, 'Engel values in residually finite groups', Monatsh. Math. 152 (2007), 169-175.

[26] P. Shumyatsky, 'Commutators in residually finite groups', Israel J. Math. 182(1) (2011), 149-156.

[27] P. Shumyatsky and J. C. Silva, 'Engel words and the restricted Burnside problem', Monatsh. Math. 159(4) (2010), 397-405.

[28] V. I. Sushchansky, 'Periodic p-elements of permutations and the general Burnside problem', Dokl. Akad. Nauk SSSR 247 (1979), 447-461.

[29] J. S. Wilson and E. Zelmanov, 'Identities for Lie algebras of pro- $p$ groups', J. Pure. Appl. Algebra 81 (1992), 103-109.

[30] E. Zelmanov, 'The solution of the restricted Burnside problem for groups of odd exponent', Math. USSR Izv. 36 (1991), 41-60.

[31] E. Zelmanov, 'The solution of the restricted Burnside problem for 2-groups', Math. Sb. 182 (1991), 568-592.

[32] E. Zelmanov, Nil Rings and Periodic Groups, Lecture Notes in Mathematics (Korean Math. Soc., Seoul, 1992).

[33] E. Zelmanov, 'Lie ring methods in the theory of nilpotent groups', in: Proceedings of Groups '93, St. Andrews, London Mathematical Society Lecture Note Series, 212 (eds. C. M. Campbell et al.) (Cambridge University Press, Cambridge, 1995), pp. 567-585.

\section{JHONE CALDEIRA, Instituto de Matemática e Estatística, Universidade Federal de Goiás, Goiânia-GO, CP 131, 74001-970, Brazil e-mail: jhone@mat.ufg.br}

PAVEL SHUMYATSKY, Department of Mathematics, University of Brasilia, Brasilia-DF, 70910-900, Brazil

e-mail: pavel@unb.br 